\title{
TB, you're a long time cured
}

\author{
James M. Trauer
}

Affiliation: School of Public Health and Preventive Medicine, Monash University, Melbourne, Australia.

Correspondence: James M. Trauer, Monash University, School of Public Health and Preventive Medicine, 553 St Kilda Road, Melbourne, Victoria 3004, Australia. E-mail: james.traueramonash.edu

@ERSpublications

Quantifying and comparing rates of chronic pulmonary aspergillosis, disease recurrence and other complications in treated TB patients are important to assess the true global impact of TB. International guidance on managing such patients would be valuable. http://ow.ly/ahQ730nHsLO

Cite this article as: Trauer JM. TB, you're a long time cured. Eur Respir J 2019; 53: 1900104 [https://doi. org/10.1183/13993003.00104-2019].

Despite official country-level estimates having been produced for nearly three decades, much remains uncertain about the true global burden of tuberculosis (TB). Official statistics focus on incidence and mortality, while health systems concentrate on supporting patients through treatment, such that less is known about the longer term damage inflicted by disease episodes.

Although its epidemiology is changing, TB remains predominantly a disease of young adulthood in many high burden countries and the organism is now the world's leading infectious killer [1]. Deaths in early adulthood often remove primary carers and main income earners from struggling households in such countries, with devastating effects for affected families. In this context, the World Health Organization's focus on avoidance of catastrophic costs and universal health coverage in the End TB era is a welcome development and should draw greater attention to TB's devastating toll [2]. However, the persisting burden of disease and debility in TB survivors is also important and under-recognised, with evidence of significant physiological impairment resulting from past TB episodes [3, 4].

Despite this, international standards do not typically provide guidance on the optimal approach to care after treatment completion $[5,6]$, such that practice is likely to vary considerably. In low to moderate burden settings, recurrent $\mathrm{TB}$ is largely attributable to relapse and is most frequent in the 2-3 years following treatment $[7,8]$, so active follow-up may be most beneficial for this period [9]. However, even during this time, rates of disease are moderate and whether active follow-up reduces diagnostic delays is unknown. Monitoring for recurrent disease is not the only rationale for considering post-treatment follow-up; post-infectious complications, such as bronchiectasis, airflow obstruction, chronic pulmonary aspergillosis (CPA) and complications of extrapulmonary disease, are all potentially important. Post-treatment follow-up provides an opportunity to target specific patient groups for tailored interventions, as well as maintaining a link between TB control services and affected communities. Patients with extensive pulmonary damage may benefit from smoking cessation interventions, pulmonary function testing and vaccination against other respiratory infections, although evidence and recommendations are again sparse.

In developing countries, recurrent $\mathrm{TB}$ is likely to occur more often because of a greater frequency of reinfection, with rates of reinfection being influenced by HIV status [10] and rates of relapse likely to vary with programmatic quality. Therefore, although the value from post-treatment follow-up is higher, resource constraints often mean that only the most effective and efficient interventions are funded. In the high burden setting of Vietnam, a recent randomised controlled trial of contact investigation found considerably higher all-cause mortality in the control arm, despite most deaths not appearing to result 
directly from TB disease itself [11]. Findings such as these are a reminder of how great the unrecognised burden of TB may be in such countries, particularly given that around one-third of all TB cases worldwide are never identified by health systems.

CPA is an important contributor to excess morbidity and mortality in TB survivors in Uganda, as described by PAGE et al. [12] in this issue of the European Respiratory Journal. Despite the logistical challenges in diagnosing a condition that has clinical, immunological, microbiological and radiological features in a resource-limited setting, the authors were successful in tracing and reassessing more than $70 \%$ of around 400 enrolled patients 2 years after completing treatment for pulmonary TB. The main findings of around 5\% and 26\% prevalence of CPA in all reassessed patients and those with cavitation, respectively, are alarmingly high, although even these figures may be under-estimates due to attrition bias. These rates are higher than my personal clinical experience in a low burden setting, where we have historically followed pulmonary patients for 2-3 years post-treatment completion [13], although they are broadly consistent with previous studies. As the authors note, the largest previous post-TB treatment cohort was described in Britain before the advent of computed tomography [14, 15], while no other study has provided such a comprehensive assessment of a cohort of this size. New diagnostic criteria for CPA were published by European Society of Clinical Microbiology and Infectious Diseases and the European Respiratory Society in 2015 [16] and by Infectious Diseases Society of America in 2016 [17], both favouring 3 months for symptom duration. The study of PAGE et al. [12] was already underway at this time, so their shorter symptom duration requirement was unavoidable, but should have little effect on their main conclusions.

Post-pulmonary TB CPA has several features that make it important clinically: it is common, treatable and has a high case fatality rate. The global prevalence of the condition can be estimated by combining data on 1) pulmonary $\mathrm{TB}$ case numbers, 2) the frequency of cavitation in surviving patients and 3) the frequency of CPA in survivors with cavitation [18]. The third quantity is probably the weakest link in this modelling approach, and has previously been estimated from the single older British study mentioned above. The data presented by PAGE et al. [12] are consistent with this historical estimate, lending weight to the previous estimates. However, the rate of CPA in the large number of patients whose TB is never diagnosed may never be known and could be systematically higher or lower than that of a post-treatment cohort.

Even when recurrent disease is diagnosed, genotypic confirmation of relapsed disease is not possible for all cases and TB recurrence rates are broadly comparable [19-21] to the rates of CPA observed by PAGE et al. [12]. Therefore, post-treatment CPA misdiagnosed as recurrent smear-negative disease could conceivably contribute a substantial proportion of reported recurrence, with implications for assessing programme performance, as well as individual patients.

Given the often severe organ damage inflicted by TB, a greater focus on patient outcomes after they have achieved satisfactory treatment outcomes is desirable in both high and low burden settings. At a minimum, further research to quantify and compare rates and predictors of post-treatment complications would help to improve the accuracy of $\mathrm{TB}$ burden estimates. Evidence-based guidance on which investigations and interventions are appropriate to which post-treatment patient groups could mitigate TB's enormous global burden, while synergies could arise from greater integration of TB care with the broader health system.

Conflict of interest: J.M. Trauer has nothing to disclose.

Support statement: This work was supported by the Department of Health, Australian Government, National Health and Medical Research Council (APP1142638). Funding information for this article has been deposited with the Crossref Funder Registry.

\section{References}

1 World Health Organization. Global Tuberculosis Report 2018. WHO/CDS/TB/2018.20. Geneva, World Health Organization, 2018.

2 World Health Organization. The END TB Strategy. WHO/CDS/TB/2018.29. Geneva, World Health Organization, 2014.

3 Hnizdo E, Singh T, Churchyard G. Chronic pulmonary function impairment caused by initial and recurrent pulmonary tuberculosis following treatment. Thorax 2000; 55: 32-38.

4 Lam KH, Jiang CQ, Jordan RE, et al. Prior TB, smoking, and airflow obstruction: a cross-sectional analysis of the Guangzhou Biobank Cohort Study. Chest 2010; 137: 593-600.

5 TB Care I. International Standards for Tuberculosis Care. 3rd Edn. The Hague, TB Care I, 2014.

6 World Health Organization. Treatment of Tuberculosis Guidelines. WHO/HTM/TB/2009.420. Geneva, World Health Organization, 2010.

7 Dale KD, Globan M, Tay EL, et al. Recurrence of tuberculosis in a low-incidence setting without directly observed treatment: Victoria, Australia, 2002-2014. Int J Tuberc Lung Dis 2017; 21: 550-555. 
8 Shen $\mathrm{X}$, Yang $\mathrm{C}$, Wu J, et al. Recurrent tuberculosis in an urban area in China: relapse or exogenous reinfection? Tuberculosis (Edinb) 2017; 103: 97-104.

9 Denholm JT, Eisen D, Fox G, et al. Management of Tuberculosis: A Guide for Clinicians. Port Adelaide, Green Hill Publishing, 2017.

10 Crampin AC, Mwaungulu JN, Mwaungulu FD, et al. Recurrent TB: relapse or reinfection? The effect of HIV in a general population cohort in Malawi. AIDS 2010; 24: 417-426.

11 Fox GJ, Nhung NV, Sy DN, et al. Household-contact investigation for detection of tuberculosis in Vietnam. N Engl J Med 2018; 378: 221-229.

12 Page ID, Byanyima R, Hosmane S, et al. Chronic pulmonary aspergillosis commonly complicates treated pulmonary tuberculosis with residual cavitation. Eur Respir J 2019; 53: 1801184.

13 Street A, McBryde ES, Denholm JT, et al. Management of Tuberculosis: A Handbook for Clinicians. Parkville, Victorian Infectious Diseases Service, the Royal Melbourne Hospital, 2012.

14 Aspergilloma and residual tuberculous cavities - the results of a resurvey. Tubercle 1970; 51: 227-245

15 Aspergillus in persistent lung cavities after tuberculosis. A report from the Research Committee of the British Tuberculosis Association. Tubercle 1968; 49: 1-11.

16 Denning DW, Cadranel J, Beigelman-Aubry C, et al. Chronic pulmonary aspergillosis: rationale and clinical guidelines for diagnosis and management. Eur Respir J 2016; 47: 45-68.

17 Patterson TF, Thompson GR, Denning DW, et al. Practice guidelines for the diagnosis and management of aspergillosis: 2016 update by the Infectious Diseases Society of America. Clin Infect Dis 2016; 63: e1-e60.

18 Denning DW, Pleuvry A, Cole DC. Global burden of chronic pulmonary aspergillosis as a sequel to pulmonary tuberculosis. Bull World Health Organ 2011; 89: 864-872.

19 Guerra-Assunção JA, Houben RMGJ, Crampin AC, et al. Recurrence due to relapse or reinfection with Mycobacterium tuberculosis: a whole-genome sequencing approach in a large, population-based cohort with a high hiv infection prevalence and active follow-up. J Infect Dis 2015; 211: 1154-1163.

20 Verver S, Warren RM, Beyers N, et al. Rate of reinfection tuberculosis after successful treatment is higher than rate of new tuberculosis. Am J Respir Crit Care Med 2005; 171: 1430-1435.

21 Luzze H, Johnson DF, Dickman K, et al. Relapse more common than reinfection in recurrent tuberculosis 1-2 years post treatment in urban Uganda. Int J Tuberc Lung Dis 2013; 17: 361-367. 